\title{
Interações medicamentosas em pacientes nefrológicos em um Hospital de Referência do Nordeste Brasileiro
}

\author{
Drug Interactions in patients with renal disorder at a Reference Hospital in Northeast Brazil \\ Interacciones medicamentosas en pacientes nefrológicos en un Hospital de Referencia \\ del Nordeste Brasileño
}

Luanna Duarte Benvindo Neiva ${ }^{1 *}$, Maria Aparecida Coelho Oliveira ${ }^{1}$, Manoel Pinheiro Lúcio Neto', Fábio Vinícius Ferreira Silva ${ }^{1}$, Mateus Cavalcante Santos ${ }^{1}$, Patrícia Nunes dos Santos ${ }^{1}$, Ernando Moreira da Costa ${ }^{1}$, Francisco Martins de Oliveira ${ }^{2}$, Josefa Natália Policarpo de Holanda ${ }^{3}$, Gilberto Portela Silva ${ }^{4}$, Deyna Francélia Andrade Próspero ${ }^{5}$.

\section{RESUMO}

Objetivo: Identificar a presença de interações medicamentosas em pacientes nefrológicos em um hospital de referência do nordeste brasileiro e verificar a frequência e gravidade dessas interações. Métodos: Trata-se de uma pesquisa de campo, de natureza descritiva, realizada a partir de análises das prescrições médicas dos pacientes nefrológicos em que foram observadas todas as prescrições médicas dos pacientes admitidos na Clínica Nefrológica, nos meses de dezembro do ano de 2018 e Janeiro do ano de 2019. As informações obtidas foram analisadas utilizando a base de dados Drugs.com e submetidas à estatística. Resultados: Das 329 prescrições analisadas, $82,98 \%$ possuíam interações medicamentosas, sendo $12,38 \%$ dessas classificadas como menores, $75,97 \%$ moderadas e $11,65 \%$ maiores. A interação medicamentosa entre Anlodipino e Enalapril (14,89\%) foi a mais frequente dentre as interações menores. As interações moderadas predominantes foram: Furosemida e Prednisona (31\%) e Anlodipino e Prednisona (30,40\%). Enquanto, nas interações classificadas como maiores prevaleceram Anlodipino e Sinvastatina (20,97\%) e Atenolol e Clonidina (10,33\%). Conclusão: É perceptível a elevada frequência de interações medicamentosas (IM) na Clínica Nefrológica, e assim, torna-se necessário lembrar a real importância da inclusão do farmacêutico na equipe multiprofissional para o sucesso terapêutico do paciente.

Palavras-chave: Paciente nefrológico, Interações medicamentosas, Farmacêutico.

\begin{abstract}
Objective: To identify the presence of drug interactions in patients with renal disorder at a reference hospital in Brazilian Northeast and to verify the frequency and severity of these interactions. Methods: This is a descriptive field study, based on analysis of the medical prescriptions in patients with renal disorders in which all the medical prescriptions of patients admitted in Nephrology Clinic were observed during December 2018 and January 2019. The information obtained was analyzed using the database Drugs.com and summarized using to statistical analysis. Results: Of the 329 prescriptions analyzed, $82.98 \%$ had drug interactions, $12.38 \%$ were classified as minor interactions, $75.97 \%$ moderate interactions and $11.65 \%$ major interactions. The drug interaction between Anlodipine and Enalapril (14.89\%) was the most frequent among the minor interactions.
\end{abstract}

${ }^{1}$ Centro Universitário Santo Agostinho (UNIFSA), Teresina - PI. *E-mail: luannadbneiva@hotmail.com

${ }^{2}$ Associação de Ensino Superior do Piauí (AESPI), Teresina - PI.

3 Universidade Federal do Piauí (UFPI), Teresina - PI.

4 Faculdade de Ciências Humanas, Exatas e da Saúde do Piauí (FAHESP/IESVAP), Parnaíba - PI.

5 Universidade Estadual do Piauí (UEPI), Teresina - PI. 
The predominant moderate interactions were: Furosemide and Prednisone (31\%), Amlodipine and Prednisone $(30.40 \%)$. While in the interactions classified as major, Anlodipine and Simvastatin (20.97\%), Atenolol and Clonidine (10.33\%) prevailed. Conclusion: The high rate of drugs interaction in the Nephrology Clinic is perceptible, and so it is therefore necessary to remember the real importance of including the pharmacist in the multiprofessional team in order to ensure therapeutic success of the patient.

Key words: Patient with renal disorder, Drug interactions, Pharmacist.

\section{RESUMEN}

Objetivo: Identificar la presencia de interacciones medicamentosas en pacientes nefrológicos en un hospital de referencia del nordeste brasileño y verificar la frecuencia y gravedad de esas interacciones. Métodos: Se trata de una investigación de campo, de naturaleza descriptiva, realizada a partir de análisis de las prescripciones médicas de los pacientes nefrológicos en que se observaron todas las prescripciones médicas de los pacientes admitidos en la Clínica Nefrológica, en los meses de diciembre del año 2018 y Enero del año 2019. La información obtenida fue analizada utilizando la base de datos Drugs.com y sometida a la estadística. Resultados: De las 329 prescripciones analizadas, 82,98\% poseía interacciones medicamentosas, siendo $12,38 \%$ de esas clasificadas como menores, $75,97 \%$ moderadas y $11,65 \%$ mayores. La interacción medicamentosa entre Anlodipino y Enalapril (14,89\%) fue la más frecuente entre las interacciones menores. Las interacciones moderadas predominantes fueron: Furosemida y Prednisona (31\%) y Anlodipino y Prednisona (30,40\%). Mientras que en las interacciones clasificadas como más grandes, prevalecieron Anlodipino y Simvastatina (20,97\%), Atenolol y Clonidina (10,33\%). Conclusión: Es perceptible la elevada frecuencia de interacciones medicamentosas (IM) en la Clínica Nefrológica, así siendo, es necesario recordar la real importancia de la inclusión del farmacéutico en el equipo multiprofesional para el éxito terapéutico del paciente.

Palabras clave: Paciente nefrológico, Interacciones medicamentosas, Farmacéutico.

\section{INTRODUÇÃO}

A utilização de vários medicamentos administrados de forma simultânea a um paciente é um ato comum na prática clínica e encontra-se relacionado diretamente ao risco de ocorrência de interações medicamentosas (IM). Entretanto, a politerapia é aceitável quando se objetiva uma terapia sinérgica ou para o tratamento de pacientes com múltiplas doenças (ROSHOLM JU, et al. 1998).

Atualmente, observa-se um grande desafio enfrentado pelos profissionais de saúde, principalmente pelos médicos, para diminuir o número de combinações de drogas na prática clínica. Nesse sentido, torna-se necessária a existência de uma estreita colaboração entre farmacêuticos e médicos clínicos para a identificação do perfil das prescrições, possibilitando assim, a troca dos medicamentos em casos nos quais a associação resulta em prejuízo ao paciente (CEDRAZ KN e JUNIOR MCS, 2014).

A gravidade das IM pode ser classificada em: maior, quando a interação pode induzir risco de vida ao paciente e/ou exigir intervenção médica para prevenir ou reduzir efeitos adversos críticos; moderada, em que a interação pode originar exacerbação da condição do doente e/ou exige uma modificação na terapia; menor, a interação limita os efeitos clínicos. Ainda nessa última, as manifestações podem proporcionar uma elevação na frequência ou severidade dos efeitos secundários, entretanto, geralmente não requer uma alteração maior na terapia (SCRIGNOLI CP et al., 2016).

A ocorrência de IMs correlaciona-se diretamente com características do fármaco, do paciente e das prescrições médicas. Os grupos de idosos, de insuficientes renais, cardíacos, hepáticos e respiratórios, com hipotireoidismo, epilepsia grave, diabetes descompensado e asma aguda, encontram-se em vulnerabilidade para a ocorrência de IMs (TEIXEIRA CC e CASTRO CGSO, 2004). No Brasil, há uma escassez de dados epidemiológicos sobre doenças renais. Além do mais, os fatores etiológicos não são bem definidos (LOPES LMV, 2001). 
Entretanto, o número estimado de pacientes que iniciaram o tratamento de diálise crônica no ano de 2016 foi de 39.714. Nesse mesmo ano, o número contabilizado de óbitos relacionados a problemas nefrológicos foi de 22.337; correspondendo assim, a uma taxa de mortalidade bruta de 18,2\% durante o ano. Nesta perspectiva, o relatório aponta uma tendência de aumento global do número de pacientes em diálise crônica, das taxas de incidência e prevalência de tratamento dialítico nos últimos anos (SESSO RC et al., 2017).

No ambiente hospitalar, as interações medicamentosas são frequentes, uma vez que o paciente faz a utilização de vários medicamentos durante a internação. A partir daí, observa-se a real importância dos profissionais conhecerem os fármacos, bem como suas interações. Principalmente, por parte dos prescritores e farmacêuticos, sendo possível assim otimizar o tratamento e reduzir os custos. Nessa perspectiva, foi de suma importância a realização do presente estudo, uma vez que, tornou-se possível alcançar o objetivo proposto pelo trabalho: identificar a presença de interações medicamentosas em pacientes nefrológicos em um hospital de referência do nordeste brasileiro, além de verificar a frequência e gravidade dessas IM. Dessa forma, o presente trabalho torna-se subsídio primordial para o reconhecimento da importância do trabalho em equipe multiprofissional em saúde no ambiente hospitalar, além disso, fornece dados para uma posterior intervenção, tornando-se uma fonte permanente de consulta para o desenvolvimento de outros trabalhos.

\section{MÉTODOS}

Trata-se de uma pesquisa de campo, de natureza descritiva, realizada a partir de análises das prescrições médicas de pacientes nefrológicos de um hospital de referência do nordeste brasileiro.

Esse hospital se localiza no centro da cidade de Teresina-PI, e destaca-se por ser um dos maiores e bem equipados hospitais da região Meio Norte do Brasil. Possui 382 leitos ativos, sendo 20 leitos reservados para pacientes da Clínica Nefrológica. Esta Unidade Clínica realiza tratamento de hemodiálise ambulatorial e diálise peritoneal, possui 11 médicos especialistas e um ambulatório para consultas.

Para realização da pesquisa foram observadas todas as prescrições médicas dos pacientes admitidos na Clínica Nefrológica, nos meses de dezembro do ano de 2018 e Janeiro do ano de 2019.

Os critérios de inclusão de análise foram todas as prescrições médicas dos pacientes internados na Clínica Nefrológica no período já citado envolvendo todas as idades, sexo e condição clínica em terapia farmacológica.

As informações obtidas no presente trabalho foram analisadas utilizando a base de dados Drugs.com (Drug Information Database), uma base abrangente e atualizada de informações sobre drogas para consulta on-line, que fornece dados gratuitos, precisos e independentes sobre mais de 24000 medicamentos.

Nesta base de dados as interações são classificadas em maiores, moderadas e menores. Os dados coletados foram analisados por meio de estatística descritiva simples, utilizando-se Microsoft Office Excel 2010 e tabulados para melhor compreensão.

Torna-se necessário lembrar que não houve abordagem direta do pesquisador com o paciente, entretanto, as análises foram realizadas de forma cuidadosa com intuito de representar com fidelidade a realidade da Clínica em estudo. A presente pesquisa foi aprovada pelo Comitê de Ética em Pesquisa com Seres Humanos (CEP) do Centro Universitário Santo Agostinho (UNIFSA), CAAE sob no 97643418.0.0000.5602.

\section{RESULTADOS}

Dentre as 329 prescrições analisadas de pacientes nefrológicos de um Hospital de referência do Nordeste Brasileiro, 17,02\% destas, o equivalente a 56 prescrições, não possuíam IM e 82,98\%, 273 prescrições, possuíam IM.

Desta forma, observou-se um total de 1648 IM nessas prescrições, sendo 204 (12,38\%) interações classificadas como menores, 1252 (75,97\%) moderadas e $192(11,65 \%)$ maiores, como apresentados no Gráfico 1. 
Gráfico 1 - Interações menores, moderadas e maiores em pacientes nefrológicos em um Hospital. TeresinaPI.

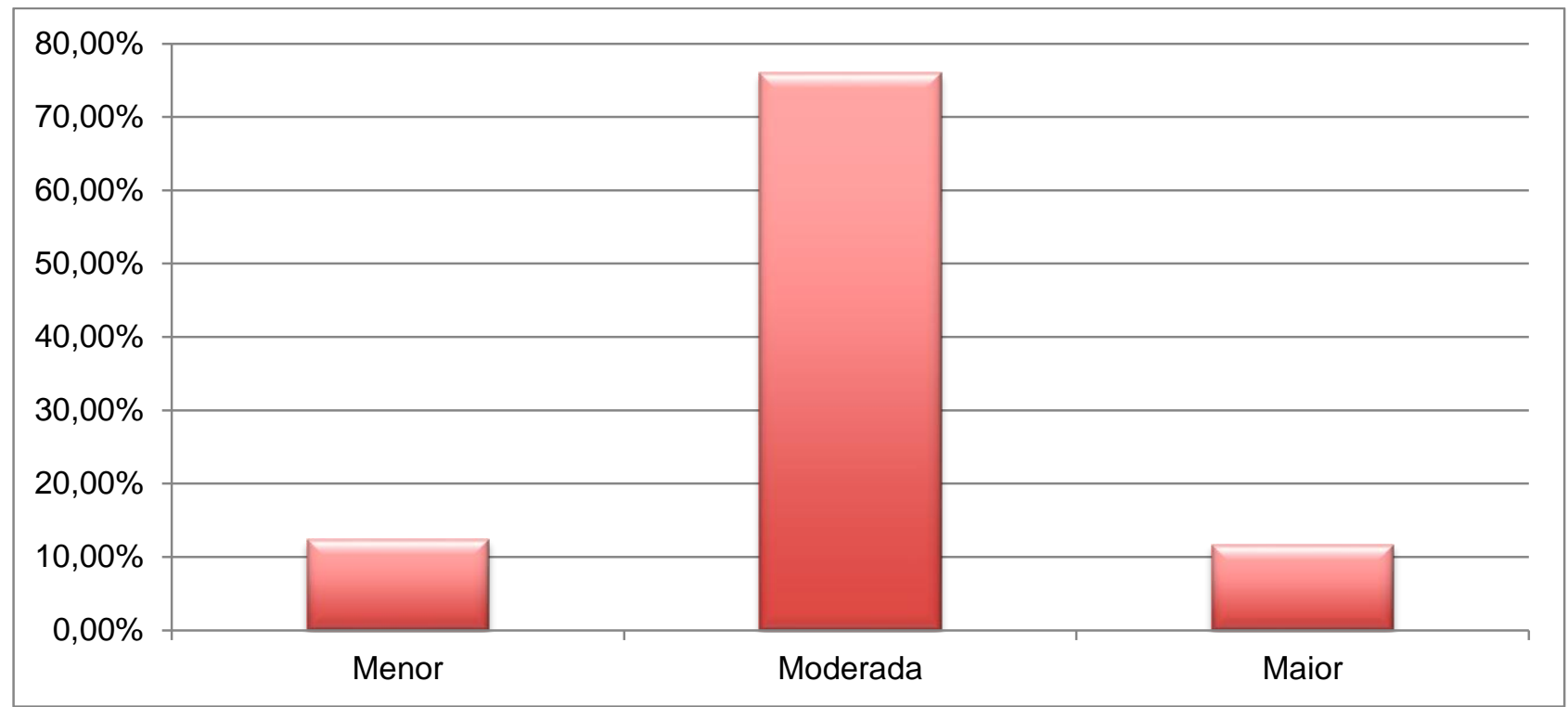

Fonte: Dados da pesquisa, 2019.

As interações classificadas como menores, de maior repetição nas prescrições analisadas, foram entre Anlodipino e Enalapril, estando presente em 49 (14,89\%) prescrições, Aspirina e Furosemida sendo evidenciadas em 15 (4,56\%) e entre Aspirina e Atenolol em 13 (3,95\%) prescrições, conforme Gráfico 2.

Gráfico 2 - IM menores mais frequentes em pacientes nefrológicos em um Hospital, Teresina-PI.

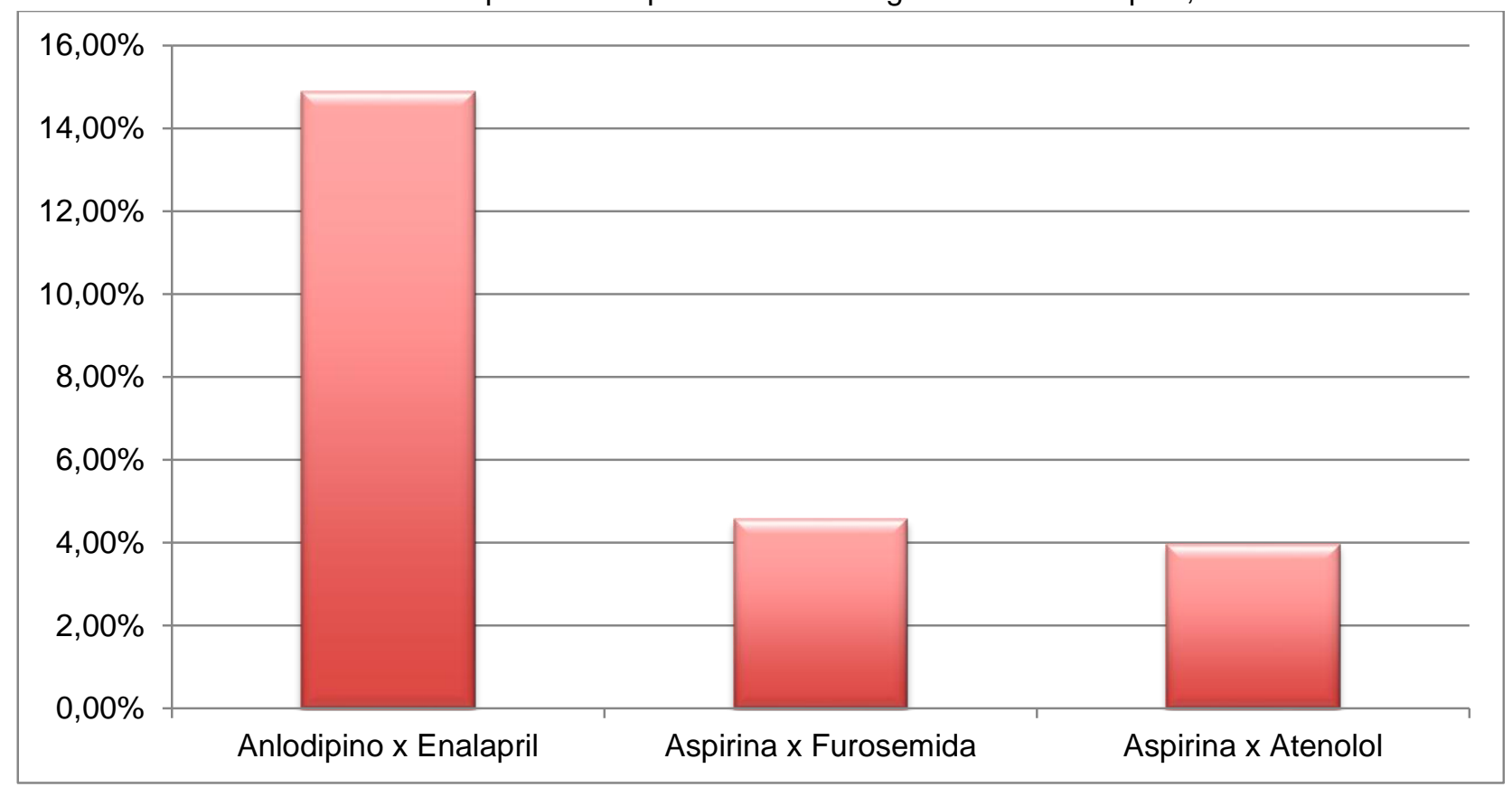

Fonte: Dados da pesquisa, 2019.

As mais frequentes interações medicamentosas, sendo classificadas como moderas, foram entre: Furosemida e Prednisona estando presente em 102 (31\%) das prescrições, seguida de Anlodipino e Prednisona existente em $100(30,40 \%)$, a terceira predominante foi entre Anlodipino e Atenolol observada em $78(23,71 \%)$ das prescrições, conforme mostra o Gráfico 3. 
Gráfico 3 - IM moderadas mais frequentes em pacientes nefrológicos em um Hospital, Teresina-PI.

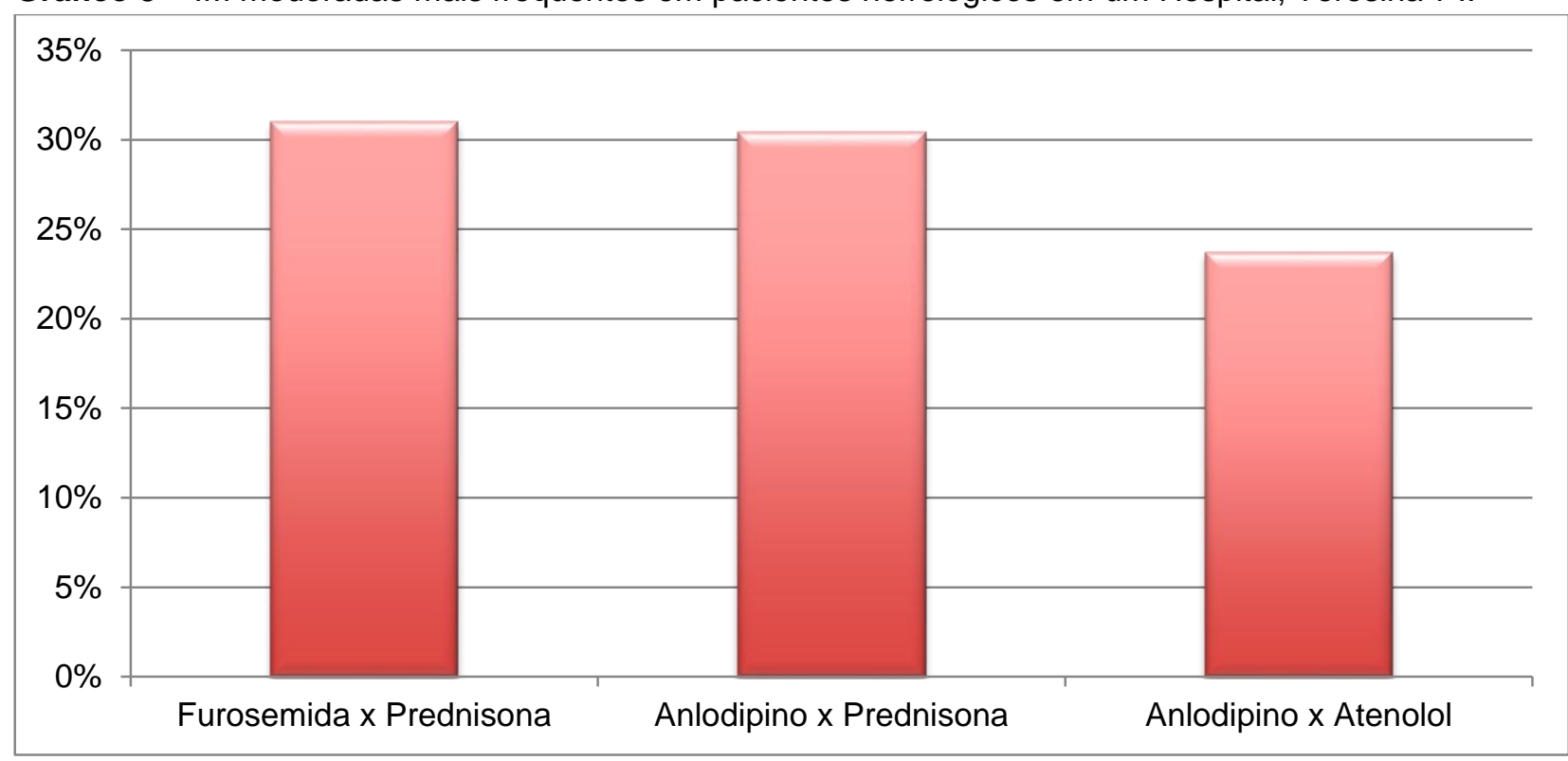

Fonte: Dados da pesquisa, 2019.

Dentre as interações classificadas como maiores, a primeira mais frequente foi entre Anlodipino e Sinvastatina presente em $64(20,97 \%)$ das prescrições, a segunda foi entre Atenolol e Clonidina, sendo evidenciada em $34(10,33 \%)$ dessas prescrições, e a terceira foi entre Ciprofloxacino e Prednisona manifestada em 15 (4,56\%) das prescrições, conforme Gráfico 4.

Gráfico 4 - IM maiores mais frequentes em pacientes nefrológicos em um Hospital, Teresina-PI.

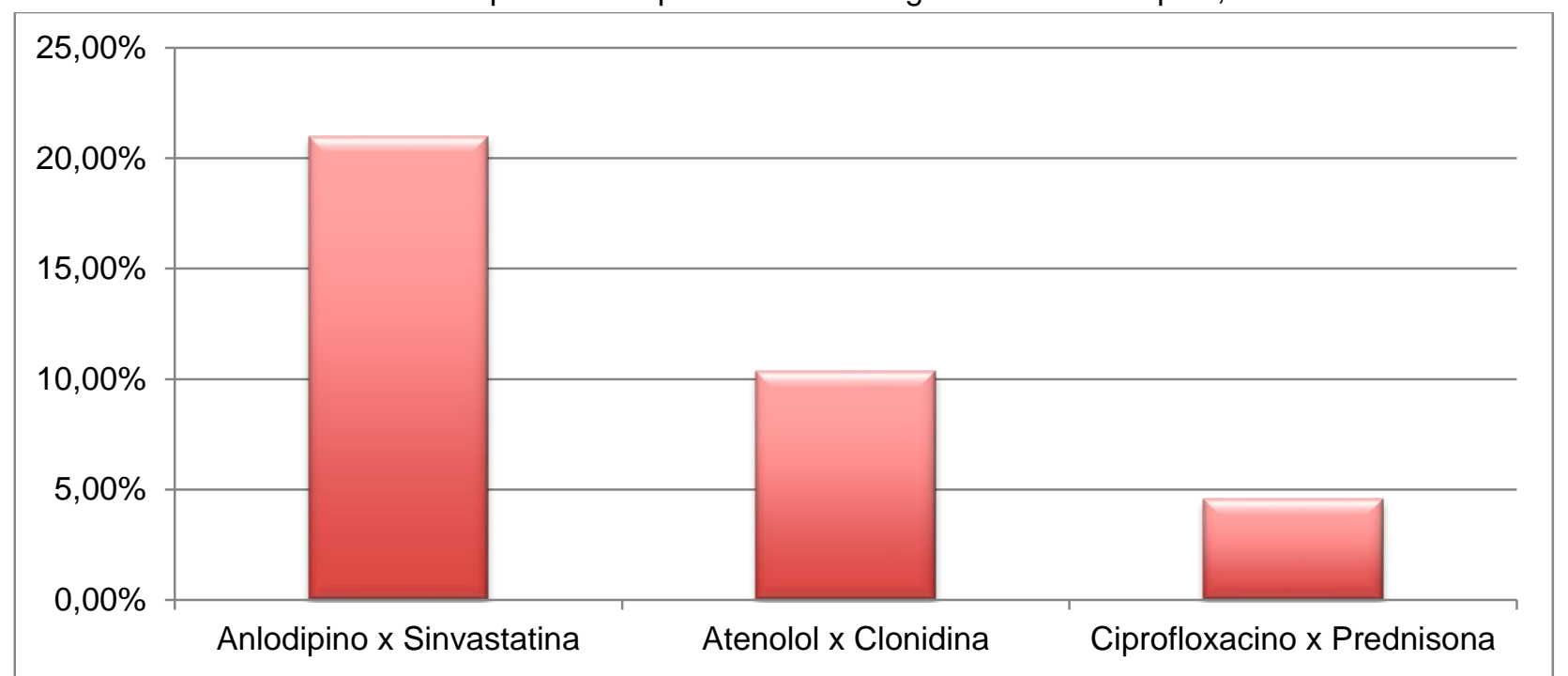

Fonte: Dados da pesquisa, 2019.

\section{DISCUSSÃO}

A interação menor predominante no presente trabalho foi entre Anlodipino e Enalapril (14,89\%), o primeiro é um antagonista de canais de cálcio (ACC) e o segundo é um inibidor da enzima conversora de angiotensina (IECA). A associação entre fármacos desses dois grupos exibe efeito sinérgico na diminuição da pressão arterial, além de proporcionar a redução do edema causado por fármacos do primeiro grupo citado (SICA DA e GEHR TWB, 2005). 
Em relação ao percentual de interações observadas entre os fármacos Aspirina e Furosemida, dentre as interações menores desse estudo, foi de $4,56 \%$ das interações. Nesse contexto, é possível observar que estes resultados contrariam o estudo de Marquito $A B$ et al. (2014) que classificou a mesma interação como sendo moderada e correspondendo a $10,10 \%$ das interações moderadas em seu trabalho, o equivalente a 106 prescrições médicas em sua amostra. O mesmo autor ainda afirma que o uso associado desses fármacos pode resultar em redução da eficácia anti-hipertensiva e diurética. A interação menor entre Aspirina e Atenolol $(3,95 \%)$ foi a terceira mais frequente dentre as interações menores neste estudo e segundo Tatro DS (2008), a atuação de um $\beta$-bloqueador na redução da pressão arterial podem ser atenuada por salicilatos; sendo assim, torna-se necessário o monitoramento do paciente que faz a utilização concomitante desses dois fármacos. Nesse caso, o prescritor pode diminuir a dose do salicilato ou alterar a terapêutica anti-hipertensiva.

Nesta pesquisa, observa-se que na Clínica Nefrológica, as IM moderadas existentes entre Furosemida e Prednisona presente em $31 \%$ das prescrições, Anlodipino e Prednisona em 30,40\%, tiveram maior prevalência nas prescrições, diferindo assim de um estudo realizado no sul da Índia em que as IM que predominaram nos pacientes com insuficiência renal, na enfermaria de nefrologia, foram entre os fármacos: ácido ascórbico e cianocobalamina em $12,45 \%$ e clonidina e metoprolol em 3,80\% das prescrições (RAMA M et al., 2012). Em outro estudo realizado em um hospital da Nigéria, em que avaliaram IMs em pacientes com doença renal crônica (DRC) foi possível verificar que as interações predominantes foram entre o lisinopril e a furosemida, furosemida e carbonato de cálcio e lisinopril e carbonato de cálcio (ADIBE MO et al., 2017).

Observa-se que as IM classificadas como maiores no presente trabalho foram a de menor ocorrência nas prescrições, ainda assim foram evidenciadas em $11,65 \%$ das interações. Torna-se necessário lembrar a necessidade da intervenção para reduzir ou prevenir graves reações adversas, uma vez que o efeito desse tipo de interação pode afetar diretamente a evolução clínica ou promover danos permanentes ao paciente caso não haja monitoração adequada do paciente (SANTOS L et al., 2013).

A interação classificada como maior, predominante neste estudo, foi a associação dos medicamentos anlodipino e sinvastatina, observada em $64(20,97 \%)$ das prescrições. Este resultado assemelha-se aos da pesquisa de Pinto NBF et al. (2014) em que as interações maiores entre estes dois fármacos também foram prevalentes em seu trabalho correspondendo à $6(15,0 \%)$ das prescrições médicas. Conforme a Food and Drug Administration (FDA, 2011), a coadministração de anlodipino e sinvastatina aumenta o risco de ocorrência de mioapatias, incluindo rabdomiólise, pois o anlodipino aumenta os níveis séricos de estatina através de modificações em sua farmacocinética, por conseguinte, torna-se necessário não exceder $20 \mathrm{mg}$ por dia de sinvastatina quando em associação com anlodipino.

Dentre as interações maiores, a segunda predominante foi entre Atenolol, um $\beta$-bloqueador, e Clonidina que estiveram presentes em 10,33\% das prescrições. Este resultado encontra-se em conformidade com o trabalho de Secoli SR et al. (2013) em que classifica a interação entre fármacos da classe dos $\beta$-bloqueadores e o ativo clonidina possuindo gravidade maior; o autor ainda explica que essa interação pode ocasionar uma hipertensão rebote no paciente, e assim recomenda alteração na terapia evitando esse tipo de interação. A terceira interação de gravidade maior, predominante nesse trabalho, foi entre Ciprofloxacino e Prednisona estando presente em $4,56 \%$ das prescrições dentre essa classificação. A administração associada de prednisona e ciprofloxacino pode ocasionar ruptura do tendão, mas o mecanismo ainda é desconhecido. Nesse sentido, torna-se necessário evitar o uso combinado desses dois fármacos em idosos, com histórico de dor, inflamação ou ruptura de tendão (OLIVEIRA HC, 2009).

Segundo Cardinal L e Fernandes C (2014), muitos erros de medicações podem ser evitados com a intervenção do profissional farmacêutico sendo realizada anteriormente à dispensação dos medicamentos; e assim, a validação da prescrição médica torna-se uma barreira visando à minimização de tais erros. Pois a dispensação só é realizada após aprovação do farmacêutico que, para um adequado funcionamento do serviço, deve ser portador de conhecimentos clínicos farmacêuticos, planejamento, banco de dados científico e atualizado, além de ter a sua disposição recursos humanos e tecnológicos. O mesmo ainda afirma que a utilização de um sistema de validação efetuada pelo farmacêutico é uma forma de garantir maior segurança e qualidade do tratamento do paciente.

REAS/EJCH | Vol. Sup. 29 | e949 | DOI: https://doi.org/10.25248/reas.e949.2019 Página 6 de 7 


\section{CONCLUSÃO}

Após a realização desse estudo é perceptível a elevada frequência de IM na Clínica Nefrológica, e assim, torna-se necessário lembrar a real importância da existência da assistência multiprofissional ao paciente, com a inclusão do profissional farmacêutico, não somente como um gestor da farmácia, mas sim como um profissional apto a realizar as intervenções farmacêuticas; contribuindo significativamente no sucesso terapêutico do paciente.

\section{REFERÊNCIAS}

1. ADIBE MO, EWELUM PC, AMORHA KC. Evaluation of drug-drug interactions among patients with chronic kidney disease in a South-Eastern Nigeria tertiary hospital: a retrospective study. Pan African Medical Journal, 2017; 28(1): 199.

2. CARDINAL L, FERNANDES C. Intervenção farmacêutica no processo da validação da prescrição médica. Rev. Bras. Farm. Hosp. Serv. Saúde São Paulo, 2014; 5(2): 14-19.

3. CEDRAZ KN, JUNIOR MCS. Identificação e caracterização de interações medicamentosas em prescrições médicas da unidade de terapia intensiva de um hospital público da cidade de feira de Santana, BA. Rev Soc Bras Clin Med, 2014; $12(2): 1-7$.

4. FDA Drug Safety Communication: new restrictions, contraindications, and dose limitations for Zocor (simvastatin) to reduce the risk of muscle injury.

5. LOPES LMV. Detecção de doenças renais: estudo populacional em um bairro da cidade de Recife-PE. Tese (Doutorado em Medicina) - Universidade Federal de São Paulo- Escola Paulista de Medicina, São Paulo, 2001; 96p.

6. MARQUITO AB, FERNANDES NMS, COLUGNATI FAB et al. Interações medicamentosas potenciais em pacientes com doença renal crônica. Jornal Brasileiro de Nefrologia, 2014; 36(1): 26-34.

7. OLIVEIRA HC. Guia prático das interações medicamentosas dos principais antibióticos e antifúngicos utilizados no Hospital Universitário Júlio Muller. Cuiabá: CIM-MT, 2009.

8. PINTO NBF, VIEIRA LB, PEREIRA FMV et al. Interações medicamentosas em prescrições de idosos hipertensos: prevalência e significância clínica. Rev enferm UERJ, Rio de Janeiro, 2014; 22(6):735-741.

9. RAMA M, VISWANATHAN G, ACHARYA LD et al. Assessment of drug-drug interactions among renal failure patients of nephrology ward in a South Indian tertiary care hospital. Indian J Pharm Sci, 2012; 74(1): 63-68.

10. ROSHOLM JU, BJERRUM L, HALLAS J et al. Polypharmacy and the risk of drug-drug interactions among Danish elderly. A prescription database study. Dan. Med. Bull, 1998; 45(2): 210-3.

11. SANTOS L, TORRIANI MS, BARROS E. Medicamentos na Prática da Farmácia Clínica. Porto Alegre: Artmed, 2013.

12. SCRIGNOLI CP, TEIXEIRA VCMC, LEAL DCP. Interações medicamentosas entre fármacos mais prescritos em unidade de Terapia Intensiva adulta. Rev. Bras. Farm. Hosp. Serv. Saúde São Paulo, 2016; 7(2): 26-30.

13. SECOLI SR, DANZI NJ, LIMA FFF et al. Interações Medicamentosas em Pacientes Coronariopatas. Rev Bras Cardiol. 2012; 25(1):11-18.

14. SESSO RC et al. Inquérito brasileiro de diálise crônica 2016. Jornal Brasileiro de Nefrologia, 2017; 39(3): 261-266.

15. SICA DA, GEHR TWB. Angiotensin-converting enzyme inhibitors. In: Oparil S, Weber MA (ed). Hypertension. Philadelphia, Pennsylvania: Elsevier Saunders; 2005. p. 669-82.

16. TATRO, DS. Drug Interaction Facts - The Authority on Drug Interactions. St. Louis. Missouri: Editora Facts \& Comparisons, 2007.

17. TEIXEIRA CC, CASTRO CGSO. Interações Medicamentosas. In FUCHS, F.D., WANNMACHER, L., FERREIRA, M.B.C. Farmacologia Clínica: Fundamentos da Terapêutica Racional. 3 Edição, Rio de Janeiro: Editora Guanabara, 2004. p. 67-72. 